\section{Antibodies to probe development}

\author{
fron John $K$. Heath
}

THE favoured method in recent years for investigating the cell surface of the early mammalian embryo has been the use of antisera raised against specific embryonic tissue, or teratocarcinoma cell lines.

The best characterised of these is probably the antiserum raised against the F9 teratocarcinoma cell line (reviewed by Jacob Immun. Rev. 33, 3; 1977). This antiserum reacts with a fairly restricted set of tissues; the preimplantation embryo, the germ line and other teratocarcinoma cell lines. The fact that a particular antiserum reacts with a particular set of tissues is not in itself very informative about the role of the cell surface in the development of the early embryo. These reagents do however provide basic information on a population of cells at the molecular level, and often show that cells which are morphologically homogeneous are unexpectedly heterogeneous in their expression of cell surface antigens. F9 antiserum, for example, reacts with nearly all F9 embryonal carcinoma cells (EC cells) but with many fewer, morphologically identical PCC4 EC cells (Seisner et al. Devl Biol. 61, 20; 1977). This and other observations are potentially important because 'classical' experimental embryology is usually based on histological analysis, and has generally classified cells into morphologically discrete types. This has led to a tendency to view the cells in a developing organism as moving through rigidly defined discrete stages. The use of antibodies not only allows one to extract more information on the effects of classical embryological manipulations, but poses questions which would never have arisen if cell morphology alone were used as a guide.

Conventional antisera can only be raised against those antigenic determinants which elicit sufficient response to produce detectable levels of antibody in the serum. Many potentially interesting molecules therefore escape detection. Conventional antisera are also often a complex mixture of antibodies directed against different determinants.

The recent development of 'hybridomas'-cell lines producing specific monoclonal antibodies - provides a way round these difficulties. If spleen cells from an immunised animal are fused with a myeloma cell line some of the resulting hybrid clones secrete antibody directed against the original immunogen (Kohler \& Milstein Nature 256,

John K. Heath is in the Department of Zoology, University of Oxford.
295 ; 1975). Each individual clone is descended from a single antibodyproducing cell in the original animal and so makes antibody directed against a single antigenic determinant. These monoclonal antibodies promise to revolutionise the study of the early mammalian embryo, amongst many other systems. They dispense with many of the technical drawbacks of conventional antisera, and allow one to find molecules hitherto undetectable on the cell surface. The first papers applying monoclonal antibodies to the study of the early mammalian embryo are now available (Stern et al. Cell 14, 775; 1978; Willison \& Stern Cell 14, 785; 1978) and many more will doubtless follow. The results of these studies again show up unexpected differences in the expression of cell surface molecules, which might not have been predicted by classical experimentation. In making hybrids between spleen cells and myelomas one is effectively sampling the whole antibody response to a complex immunogen, and it is therefore possible to make antisera directed against very minor determinants in a population of cells. Indeed, the original immunogen used by Stern et al. was a preparation of lymphocytes, partially enriched for $T$ cells. One of the clones resulting from the fusion made a product which reacted with a minor subpopulation of lymphocytes (about $2 \%$ ) but reacted with EC cells to a much greater extent. This illustrates the value of screening the products of hybrids against a panel of cell types other than the original immunogen. Further investigation showed that the antibody produced by this particular clone reacted with sheep red blood cells, brain and kidney as well as with some somatic component of the testes. The species and tissue distribution suggested that this antigen was similar to Forssman antigen, and the case for this was strengthened by the observation that the antigenic determinant was carried on a glycolipid molecule.

When attention was turned to the preimplantation embryo however, the distribution of this single determinant turned out to be most unusual. The determinant first appeared on the late morula stage (16-32 cells), but could not be found on all embryos. The amount of staining increased as the trophectoderm formed, but again not all trophectodermal cells reacted with the antiserum, and there was no obvious pattern of reactivity common to all embryos. In some cases it appeared as though only one or two cells in the trophectoderm carried the determinant. If the embryo was allowed to grow out in culture (mimicking the process of implantation) the determinant disappeared from the trophectoderm completely.

The strange distribution of this determinant does not fit in with any preconceived notions of trophectodermal formation or differentiation. It is possible that the expression of this determinant is related to the cell cycle, or that the molecule is perhaps somehow masked in the cell membrane. In any case these findings call for a number of experiments which would not even have been conceived or made possible without antibodies.

Many embryologists believe that the cell surface has an important role in development. There is, unfortunately, very little direct evidence to comfort them. Perhaps monoclonal antibody technology will not only stimulate experiments to reassure the faithful, but help to challenge a number of long held beliefs about developmental proceses in general.

\section{History of biology at the Naples Zoological Station}

from Robert Olby

FEw biologists can be unaware of the important contribution which the Stazione Zoologica di Napoli has made over the years since the first visiting scientist came to work there in 1873 . As Theodor Boveri declared--."How many prosperous investigations, how much joy of discovery, has this house known!" Established by the wealthy German zoologist and student of the origin of the vertebrates, Anton Dohrn, the station was intended to foster internationally the study of marine organisms. As an enthusiast for the Darwinian theory, Dohrn believed that comparative studies of the development of higher and lower marine organisms would provide the key to the phylogeny of the vertebrates. In fact the Station has been the site for researches of very wide significance in a variety of fields. They include Boveri's classic researches into nucleo-cytoplasmic relations and the individuality of the chromosomes in the sea urchin egg, and Otto Warburg's measurements of the respiration rate of eggs before and after fertilisation, a landmark in the introduction of physiology into development biology. Others who carried out researches at the Station were

* Held at the Naples Zoological Station Annexe on the island of Ischia from July 1-15. 